\title{
An electromyographic assessment pilot study on the reliability of the forearm muscles during multi-planar maximum voluntary contraction grip and wrist articulation in young males
}

Henry H. Hunter

Graeme G. Sorbie

Fergal M. Grace

Yaodong Gu

Wing-Kai Lam

Julien S. Baker

Frédéric Dutheil

Tilak Dias

Ukadike C. Ugbolue

This is the accepted manuscript published in Technology and Health Care. The definitive and edited version of the article is available online at DOI:

http://dx.doi.org/10.3233/THC-212822

Hunter, H.H., Sorbie, G.G., Grace, F.M., Gu, Y., Lam, W.K., Baker, J.S., Dutheil, F., Dias, T. \& Ugbolue, U.C. (2021) 'An electromyographic assessment pilot study on the reliability of the forearm muscles during multi-planar maximum voluntary contraction grip and wrist articulation in young males'. Technology and Health Care. 


\section{Technology and Health Care}

Title:

An Electromyographic Assessment Pilot Study on the Reliability of the Forearm Muscles during MultiPlanar Maximum Voluntary Contraction Grip and Wrist Articulation in Young Males.

\section{Author names and affiliations:}

Henry H. Hunter ${ }^{a, b}$, Graeme G. Sorbiec, Fergal M. Grace, ${ }^{d}$, Yaodong Gu', Wing-Kai Lam ${ }^{\text {eff }}$, Julien S. Baker $^{\mathrm{a}, \mathrm{g},}$, h,i Frédéric Dutheil, 'Tilak Dias and Ukadike C. Ugbolue ${ }^{\mathrm{a}, \mathrm{b}, \mathrm{k}}$

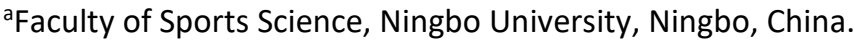

${ }^{b}$ School of Health and Life Sciences, Institute for Clinical Exercise \& Health Science, University of the West of Scotland, Lanarkshire, G72 OLH, United Kingdom.

'Division of Sport and Exercise Sciences, School of Applied Sciences, Abertay University, Dundee, DD1 1HG, United Kingdom.

${ }^{\mathrm{d}}$ Faculty of Health, Human Movement \& Sport Sciences, Federation University Australia, Ballarat, Victoria, Australia.

eLi Ning Sports Science Research Center, Li Ning (China) Sports Goods Co. Ltd, Beijing, China.

fDepartment of Kinesiology, Shenyang Sports Institute, Shenyang, China.

${ }^{g}$ Centre for Health and Exercise Science Research, Department of Sport, Physical Education and Health, Hong Kong Baptist University, Kowloon Tong, Hong Kong.

${ }^{h}$ CNRS, LaPSCo, Physiological and Psychosocial Stress, University Hospital of Clermont-Ferrand, CHU ClermontFerrand, Preventive and Occupational Medicine, WittyFit, Université Clermont Auvergne, 63000 ClermontFerrand, France.

'Faculty of Health, School of Exercise Science, Australian Catholic University, Melbourne, VIC 3000, Australia.

'Advanced Textiles Research Group, School of Art and Design, Bonington Building, Nottingham Trent University, UK

kDepartment of Biomedical Engineering, University of Strathclyde, Glasgow, United Kingdom.

\section{Corresponding author:}

Ukadike Chris Ugbolue,

Biomechanics Laboratory,

School of Health and Life Sciences,

Institute for Clinical Exercise \& Health Science,

University of the West of Scotland, Lanarkshire, G72 OLH, United Kingdom.

Phone: +44 (0)1698 283100 Ext 8284

Email: u.ugbolue@uws.ac.uk 


\section{Abstract}

BACKGROUND: Electromyographic systems are widely used in scientific and clinical practice. The reproducibility and reliability of these measures are crucial when conducting scientific research and collecting experimental data.

OBJECTIVE: To test the reliability of surface electromyography signals from both the Flexor Digitorum Superficialis (FDS) and Extensor Carpi Radialis Brevis (ECRB) muscles of both the left and right arms during an individual, static multi-planar maximum voluntary contraction handgrip task using the Myon 320 system (Myon AG, Switzerland).

METHODS: Eight right-handed male participants performed two maximal handgrip tests in five separate wrist positions using both hands. Muscle activity was recorded from both forearms. Reliability was measured using the Standard Error of Measurement (SEM), Coefficient of Variation (CV) and Intra-class correlation coefficients. Wrist joint position correlations within and between the FDS and ECRB muscle activities were also analysed.

RESULTS: Absolute reliability was shown across all positions for both hands with CV and SEM recorded at below 10\%. The output measures indicate that the Myon 320 system (Myon AG, Switzerland) produces good to fair reliability when assessing forearm muscle activity. Correlations in the left FDS muscles were negative. Correlations between the left ECRB and left FDS muscles were variable but positive between the right ECRB and right FDS muscles.

CONCLUSIONS: The data sets retrieved from all participants were reliably evaluated. Wrist position correlations within and between the FDS and ECRB muscles may have been influenced by hand dominance. The findings demonstrate that the methods and systems outlined in this study can be used reliably in future research.

Keywords: EMG; Consistency; Hand Grip; FDS; ECRB 


\section{Introduction}

Snijders and associates point out that both the finger flexors, the wrist and finger extensors are active during grasping and pinching and that this activity intensifies with increases in grasping or pinching force [1]. Handgrip strength testing is a method widely employed in various sporting contexts and is also used by occupational therapists in a range of clinical settings, particularly in the investigation of hand pathologies and associated injuries [2]. Common ways to assess hand function is to measure grip strength and pinch strength [3]. Grip strength has become a reliable and practical method to measure human strength [4]. The methodology is widely used in adults as an indicator of strength assessment utilised in fitness testing and readiness for participation in sports [5]. Tietjen-Smith and colleagues found a direct correlation between grip strength and overall body strength in very elderly females [6]. Fry and co-researchers found a correlation between grip strength and performance in American male junior weightlifters [7].

However, Budoff and associates confirmed that grip strength may be influenced by many other variables including nutritional status, weakness in the rotator cuff, fatigue and overall physical function [8]. Many daily active movements and sporting skills depend on the high activity levels of the extensor and flexor musculature of the forearms and hands, which can influence grip strength. This indicates that the strength of an athlete's grip can be a key factor in performance, injury prevention and overall strength assessment of developing athletes [9]. The kinematic characteristics of the wrist are obtained by analysing two significant planar motions of the hand relative to the forearm, these being the flexion-extension motion and radial-ulnar deviation. The functional range of motion, the looseness locus, and the instantaneous centre of rotation locus are the three mechanical measures used to quantify the kinematic behaviour of the wrist [10]. In addition, Hoffman and associates highlight that the kinematic movement of the wrist is also centred around radial and ulnar deviation, extension and flexion [11]. 
Intermittent hand gripping effort is also frequently used by industrial workers in addition to athletes for the evaluation of the potential to perform various manual employment activities [12]. In the last seven years, only a few studies have been published that evaluate hand muscle function from an electromyographic (EMG) perspective. Examples of these include: Case studies using EMG to record muscle activities of a motorcyclist [13]; EMG assessments based on hand size, grip force and stroke rotation during screw driving [14]; analyses of muscle activation during pull ups [15] and a study investigating the use of EMG to evaluate recumbent hand cycling [16].

An earlier study by Goh and colleagues investigated the effects of sleep deprivation on hormonal profiles and handgrip strength performance [17]. They found that grip performance increased during the day and declined at night and that sleep deprivation lowered performance levels. This demonstrates that grip strength can be affected by diurnal variations as well as the quantity and quality of rest [18]. Hoozemans et al point out that the EMG of the Flexor Digitorus Superficialis (FDS) may be useful to predict grip force [19]. Meanwhile, Chen and colleagues have produced results to verify that test-retest values obtained from a pinch grip test can be used successfully on the nonaffected arms of stroke patients [20].

Neither of these studies used the Myon 320 system (Myon AG, Switzerland) for electromyographic data capture, however, a study by Sorbie et al (2018) investigated the Myon systems (Myon AG, Switzerland) reliability during a bilateral front raise, a squat test and an isometric handgrip task [21]. The portability, ease of use and compatibility in conjunction with the Vicon Motion Analysis System allows users to not only examine muscle output during movement, but also to track and map movement patterns of specific anatomical points within a three-dimensional area. Incel et al (2002) state that the reliable and valid evaluation of hand strength is of great importance in determining the effectiveness of different treatment strategies or for evaluating the effects of different hand related experimental and surgical procedures [22]. To date several studies have been completed that utilised surface Electromyography (sEMG) on many different muscle groups, however, there remains only a 
small number of studies that have measured and validated forearm muscle activity using the Myon 320 system (Myon AG, Switzerland).

The purpose of this investigation, therefore, was to further evaluate the reliability of the Myon 320 system (Myon AG, Switzerland) when capturing electromyographical output from two forearm muscles (Extensor Carpi Radialis Brevis (ECRB) and Flexor Digitorum Superficialis (FDS)) that are active during a gripping task.

\section{Method}

\section{Participants}

Eight right-handed male participants volunteered to take part in this laboratory-based pilot study. (Age: $22.5 \pm 7.6$ years, Height: $185.5 \pm 5.40 \mathrm{~cm}$, Weight: $82.18 \pm 29.69 \mathrm{~kg}$ ). Participants had no wrist or elbow injuries within a twelve-month period and no hand, wrist or elbow surgeries in the previous five years. Prior to testing, participants were required to avoid any form of physical activity for at least 24 hours before their designated trial start time. All participants were tested at the same time of day to minimize potential diurnal effects on data capture. A Par-Q and consent form was also completed by the participants. Ethical approval was granted by the University of the West of Scotland Ethics Committee. All participants signed an informed consent form outlining the purpose of the experiment and stating that participants were free to withdraw from the study at any time.

\section{Apparatus}

A Myon 320 sEMG System (Myon AG, Switzerland) was used with AMBU surface electrodes to capture the output of the ECRB and FDS during each Maximum Voluntary Contraction (MVC). The Myon 320 sEMG system (Myon AG, Switzerland) was interfaced with a Vicon Bonita Motion Analysis System (Oxford, UK). The motion capture system was used to monitor the markers placed on the elbow joint 
and segments of the forearm and upper arm. Three markers were placed on the forearm, elbow joint and upper arm. This ensured that the forearm and upper arm at the elbow joint remained at $90^{\circ}$ for all participants during the experiment. A Medical Research Ltd (Leeds, UK) pinch / grip digital analyzer with winCAS software was used to capture the force $(\mathrm{N})$ of each maximal hand grip for a maximum time period of five seconds.

\section{EMG Procedures}

Prior to testing the participants' skin was prepared for placement of the EMG AMBU surface sensors (AMBU, Cambridge, UK). This process involved hair removal from the specific areas with a disposable razor, followed by rubbing with a fine abrasive AMBU Skin Prep Pad for skin preparation (AMBU, Cambridge, UK) before thoroughly cleaning with a hypoallergenic alcohol swab (AMBU). Surface sensors were then placed on the FDS and ECRB forearm muscles before connection to the electrode transmitters. When measuring muscle activity for the ECRB muscles during the current study an interelectrode distance of $2 \mathrm{~cm}$ was selected in accordance with previous experimentation.[23] For the ECRB muscle, a line was marked between the lateral epicondyle and the radial styloid process. The ECRB is located at the proximal half of the forearm, just lateral to the line.[24] The electrode for the FDS muscle was placed towards the middle of the forearm, halfway from the ventral midline to the medial border of the forearm. There was no risk of crosstalk with respect to the placement of the EMG AMBU surface sensors (AMBU, Cambridge, UK) on the muscle bellies of the FDS and ECRB muscles. Each subject adopted a standardized sitting position with the arm attached to a custom rig and with the elbow flexed at $90^{\circ}$ during the hand grip test (Figure 1). 


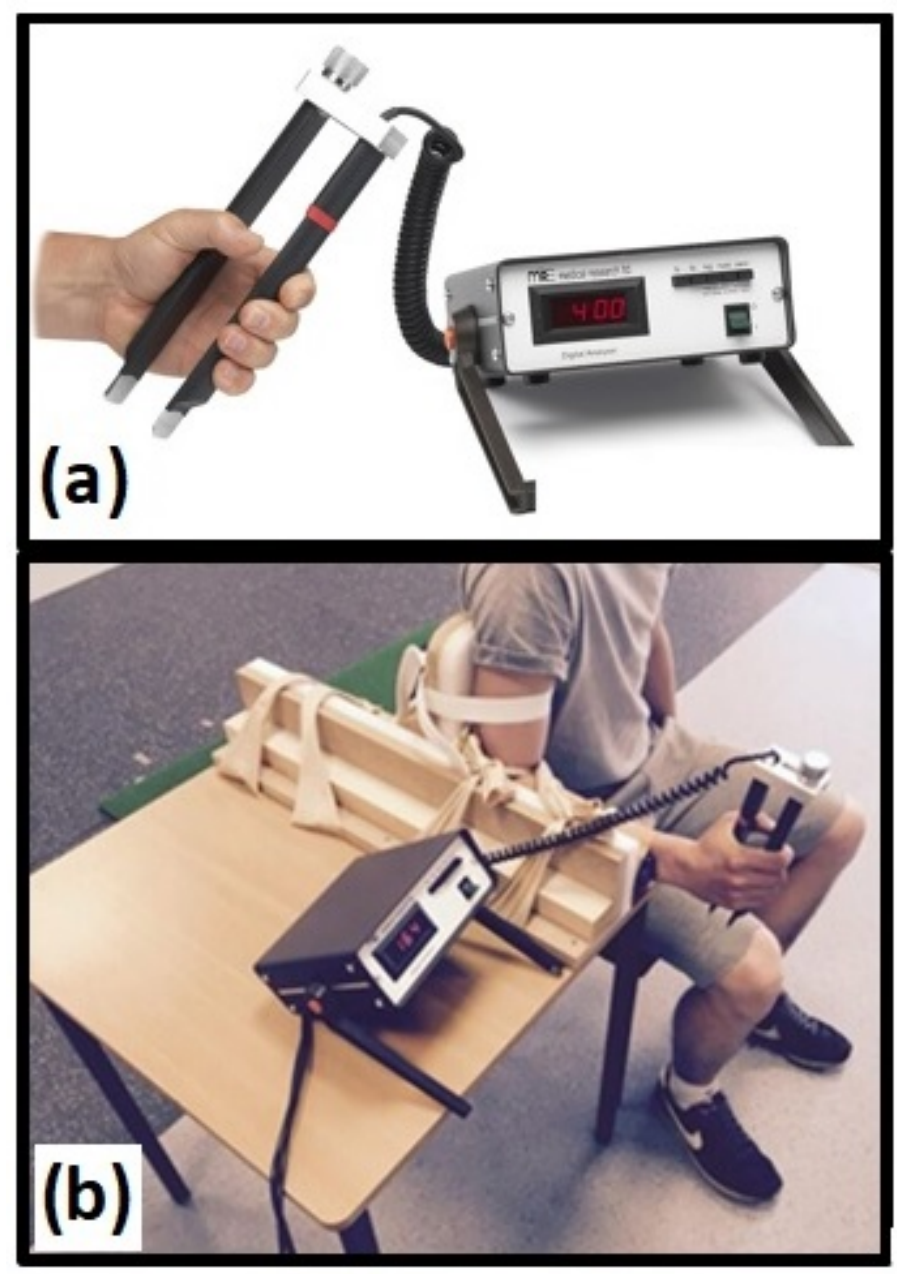

Figure 1. (a) Pinch / Grip Digital Analyzer Device (b) Pictorial illustration of a subject using Pinch / Grip Digital Analyzer Device with forearm secured in a customized arm rig.

\section{Experimental Design}

Prior to data collection all procedures were explained to participants and all were familiarized with testing protocols. The dynamic warm up session was unnecessary for this pilot study for fear of fatiguing the FDS and ECRB muscles before the handgrip task was performed. Each participant performed three maximal handgrips in five separate randomized positions (Neutral, Flexion, Extension, Radial Deviation and UInar Deviation) for a maximum of five seconds with a two-minute rest period between efforts. Verbal stimulations to the volunteers during the maximal contractions 
were provided. This procedure was then repeated for the other hand. A Medical Research Ltd (Leeds, UK) pinch / grip digital analyzer was used to capture the force $(\mathrm{N})$ of each maximal handgrip for a maximum time period of five seconds. A total of fifteen trials were carried out for each hand using five separate wrist positions (Neutral, Flexion, Extension, Radial Deviation and Ulnar Deviation) and three repetitions per position.

\section{Data Analysis}

Data was captured at a frequency of $1000 \mathrm{~Hz}$ with the implementation of a high/low pass Butterworth filter (High $20 \mathrm{~Hz}$, Low $400 \mathrm{~Hz}$ ) to reduce any transient noise before Root Mean Square (RMS) (20ms) values were calculated. These procedures followed methodologies outlined in a previous study [21]. All data were exported to Microsoft Excel 2016 (Microsoft Corporation, Redmond, Washington) and analysed using the IBM Statistical Package for Social Sciences (SPSS) Statistics for Windows, Version 25.0 (IBM Corp., Armonk, New York). Descriptive and inferential statistics were also incorporated.

A Shapiro-Wilks test was applied to the dataset to examine if the data was normally distributed. A paired t-test was used to establish if there were any statistical differences between left and right forearms with respect to each the FDS and ECRB muscles. Pearson correlation $(r)$ was performed to establish the level of muscle activity interaction between the FDS and ECRB muscles within and between both hands. When implementing the Pearson correlation, the $r$ values obtained varied between -1 and +1 where 1 is a perfect correlation and 0 represents no correlation. Further interpretations of $r$ include: $1>r \geq 0.8$ (Very Strong); $0.8>r \geq 0.6$ (Moderate); $0.6>r \geq 0.3$ (Fair) and $0.3>r \geq 0.1$ (Poor) $[25,26]$. Reliability was assessed based on studies by Atkinson and associates who suggested that a measurement tool is reliable if the Intraclass Correlation Coefficient (ICC) is above 0.800 and $\mathrm{CV}$ is below $10 \%$ [27]. A mixed model and absolute type ICC was run. SEM was also used to express absolute reliability of the measure. A measure of internal consistency to evaluate the scale of reliability was also determined using Cronbach's alpha $(\alpha)$ [28]. The scale was interpreted as follows: 
If $\alpha \geq 0.9$ (Excellent), $0.9>\alpha \geq 0.8$ (Good), $0.8>\alpha \geq 0.7$ (Acceptable), $0.7>\alpha \geq 0.6$ (Questionable), 0.6 $>\alpha \geq 0.5$ (Poor), $0.5>\alpha$ (Unacceptable). The CV and the SEM displayed in equations (1) and (2) were calculated using the equations presented below at $95 \%$ confidence interval $(\mathrm{Cl})$ :

$$
\begin{aligned}
& C V=\frac{\mathrm{SD}}{\text { Mean }} \times 100 \% \\
& \operatorname{SEM}(x)=\frac{S D}{\sqrt{N}}
\end{aligned}
$$

Where SD is the standard deviation and $\mathrm{N}$ refers to the sample size.

A repeated measures ANOVA was used to evaluate differences recorded for the FDS and ECRB muscles respectively with respect to the five wrist positions. To avoid any variation from trial to trial the MVC's of each wrist position (Extension, Flexion, Radial Deviation and Ulnar Deviation) were averaged and then calculated as a percentage of the normalized dataset (Neutral mean for both arms). Effect sizes were evaluated using partial ETA squared statistic $\left(\eta_{p}{ }^{2}\right)$ in relation to the ANOVA. The threshold values of $0.0099,0.0588$, and 0.1379 were considered small, medium, and large effect sizes respectively [29]. Throughout the study the significance level was set at $P=0.05$.

\section{Results}

All participants displayed similar trends when recording readings from the EMG outputs for both forearm muscle groups for the five measurements on both arms. On average, the greater muscle activation was displayed by the right ECRB during the Ulnar Deviation wrist position (134.92\%). The highest left FDS activation figure was also shown during Ulnar Deviation (100.96\%). Minimal activation was shown to be from the left FDS when in the Radial Deviation position (72.78\%). Similarly, to the pattern shown with the left FDS, Ulnar Deviation of the right FDS also proved to produce the lowest 
output for that side. When comparing the activation of the FDS and ECRB for Extension, Flexion, Ulnar Deviation and Radial Deviation the right ECRB across all wrist positions (128.74\% (Flexion); $111.97 \%$ (Radial Deviation); $134.92 \%$ (Ulnar Deviation)), displayed greater activity with exception of the right FDS (101.36\%) against the right ECRB (97.89\%) during Extension (Table 1).

Table 1. Comparative percentage against Neutral.

\begin{tabular}{lllll}
\hline Wrist Position & Left FDS & Right FDS & Left ECRB & Right ECRB \\
\hline Extension & $79.86 \pm 29.32$ & $101.36 \pm 46.24$ & $87.64 \pm 25.23$ & $97.89 \pm 46.03$ \\
Flexion & $95.54 \pm 37.50$ & $115.48 \pm 57.38$ & $117.40 \pm 34.22$ & $128.74 \pm 73.88$ \\
Radial Deviation & $72.78 \pm 38.87$ & $99.57 \pm 57.53$ & $98.66 \pm 44.45$ & $111.97 \pm 60.99$ \\
Ulnar Deviation & $100.96 \pm 44.59$ & $122.11 \pm 57.24$ & $120.65 \pm 52.42$ & $134.92 \pm 69.00$ \\
Neutral & $100.00 \pm 0.00$ & $100.00 \pm 0.00$ & $100.00 \pm 0.00$ & $100.00 \pm 0.00$ \\
\hline
\end{tabular}

A comparison of the muscle activation of the wrist for both arms. The Radial and Ulnar Deviation showed a greater output for both Left/Right FDS and ECRB when in Ulnar Deviation. The Ulnar Deviation wrist position produced the highest muscle activation output (Table 1). The Flexion position produced the greatest output when compared to Extension (95.54\% (Left FDS); 115.48\% (Right FDS); $117.40 \%$ (Left ECRB); $128.74 \%$ (Right ECRB)). Flexion also produced greater percentage muscle activation when compared to the wrist in Radial Deviation (Table 1).

Intra-session ICC, showed excellent reliability (>0.9) in extension for the left ECRB, right FDS and right ECRB, $(95 \% \mathrm{Cl}=0.905-0.998),(95 \% \mathrm{Cl}=0.741-0.987),(95 \% \mathrm{Cl}=0.883-0.994)$ as well as right $\mathrm{ECRB}$ flexion (95\% Cl $=0.990-0.998)$ and radial deviation for left FDS (95\% Cl $=0.659-0.983)$, left and right ECRB $(95 \% \mathrm{Cl}=0.894-0.994),(95 \% \mathrm{Cl}=0.741-0.986)$. Good reliability $(0.75-0.9)$ was achieved in extension by the left FDS $(95 \% \mathrm{Cl}=0.540-0.974)$, right flexion $(95 \% \mathrm{Cl}=0.103-0.954)$, and radial deviation $(95 \% \mathrm{Cl}=0.501-0.975)$, left ECRB flexion, neutral $(95 \% \mathrm{Cl}=0.345-0.965),(95 \% \mathrm{Cl}=0.107-$ $0.955)$ and right ECRB neutral $(95 \% \mathrm{Cl}=0.419-0.972)$. Ulnar deviation produced the poorest overall results along with left FDS in flexion and right FDS in the neutral positions, $(95 \% \mathrm{Cl} \leq 0.7)$. 
Table 2. Muscle activation outputs and reliability measurements of the ECRB and FDS muscles in five wrist positions.

\begin{tabular}{lllllll}
\hline $\begin{array}{l}\text { Muscle } \\
\text { Activation }\end{array}$ & $\begin{array}{l}\text { Reliability } \\
\text { Measurement }\end{array}$ & Extension & Flexion & Neutral & $\begin{array}{l}\text { Radial } \\
\text { Deviation }\end{array}$ & $\begin{array}{l}\text { Ulnar } \\
\text { Deviation }\end{array}$ \\
\hline Left FDS & SD & $29.32 \%$ & $37.50 \%$ & $100.00 \%$ & $38.87 \%$ & $44.59 \%$ \\
& SEM & $3.31 \%$ & $4.24 \%$ & $11.31 \%$ & $4.39 \%$ & $5.04 \%$ \\
& CV & 0.17 & 0.48 & 0.38 & 0.36 & 0.25 \\
Right FDS & SD & $49.43 \%$ & $61.34 \%$ & $100.00 \%$ & $61.51 \%$ & $61.20 \%$ \\
& SEM & $5.59 \%$ & $6.94 \%$ & $11.31 \%$ & $6.95 \%$ & $6.92 \%$ \\
& CV & 0.85 & 0.79 & 0.59 & 1.00 & 0.86 \\
Left ECRB & SD & $26.97 \%$ & $36.58 \%$ & $100.00 \%$ & $47.52 \%$ & $56.04 \%$ \\
& SEM & $3.05 \%$ & $4.14 \%$ & $11.31 \%$ & $5.37 \%$ & $6.34 \%$ \\
& CV & 0.39 & 0.26 & 0.34 & 0.43 & 0.27 \\
Right ECRB & SD & $52.11 \%$ & $70.09 \%$ & $100.00 \%$ & $56.48 \%$ & $61.72 \%$ \\
& SEM & $5.89 \%$ & $7.93 \%$ & $11.31 \%$ & $6.39 \%$ & $6.98 \%$ \\
& CV & 0.51 & 0.72 & 0.64 & 0.52 & 0.59 \\
\hline
\end{tabular}

Standard Deviation (SD), Standard Error of Measurement (SEM) and Coefficient of Variation (CV).

Table 2 shows a greater error variance from the right FDS and ECRB when compared to the left. However, this is marginal, and all data from both SEM and CV display a reliability figure of less than 10\%. Reliability was also measured by calculating Cronbach's Alpha of the percentage of neutral data. This gave an output of 0.987 to further confirm the reliability of the tests.

In addition to the reliability results reported above, a paired t-test was performed and clearly identified that there was no significant difference $(P>0.05)$ in EMG output between the left and right FDS muscles and the left and right ECRB muscles. There was however a significant difference $(P<0.05)$ when comparing the FDS muscle against the ECRB muscle on the same arm.

The repeated measures ANOVA revealed no significant differences $\left(P=0.604, F=0.560, \eta_{p}^{2}=0.038\right.$, small) between FDS muscle activity for both the left and right hands with respect to all the wrist joint positions. In addition, there were no significant differences ( $P=0.967, F=0.064, \eta_{p}{ }^{2}=0.005$, small) between the ECRB muscle activity for the left and right hands respectively with respect to all the wrist 
positions. The within muscle activity correlations with regards to the left or right hand wrist positions are shown in Figure 2. Within the left FDS muscle, the wrist position correlation produced a negative correlation ranging from -0.06 (Poor) to -0.33 (Fair) between extension and the four other wrist positions. Correlations between ulnar deviation and flexion $(r=-0.04$ (Poor)) and ulnar deviation and neutral ( $r=-0.26$ ((Poor)) were also negatively correlated for the FDS muscle. The left FDS also exhibited a positive correlation for the flexion and neutral ( $r=0.61$ (Moderate)) and ulnar deviation and radial deviation ( $r=0.48$ (Fair)) associations. Aside from both the ulnar deviation and extension correlation ( $r=-0.55)$ and ulnar deviation and radial deviation ( $r=-0.34$ (Fair)), all other correlations were positive (Figure 2). Both the right FDS and ECRB muscle activities produced Fair to Very Strong positive correlations $(1>r \geq 0.3)$ for all wrist position associations. Apart from the left FDS ulnar deviation and radial deviation wrist position correlation $(P=0.019)$ all other left FDS wrist position correlations were not significant $(P>0.05)$. No significant differences were observed for all left ECRB wrist position associations. The right FDS produced only a significant difference between ulnar deviation and radial deviation associations $(P=0.025)$. The right ECRB displayed significant differences for the ulnar deviation and extension association $(P=0.044)$ and ulnar deviation and radial deviation association $(P=0.043)$.

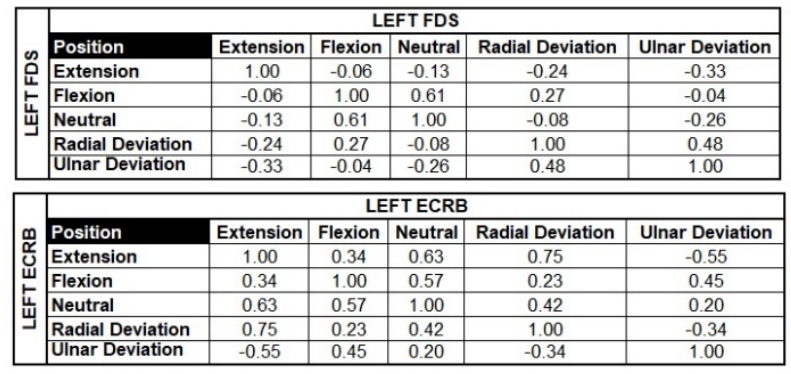

\begin{tabular}{|c|c|c|c|c|c|c|}
\hline \multirow{7}{*}{ 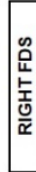 } & \multicolumn{6}{|c|}{ RIGHT FDS } \\
\hline & Position & Extension & Flexion & Neutral & Radial Deviation & Ulnar Deviation \\
\hline & Extension & 1.00 & 0.60 & 0.73 & 0.95 & 0.92 \\
\hline & \begin{tabular}{|l|} 
Flexion \\
\end{tabular} & 0.60 & 1.00 & 0.33 & 0.70 & 0.81 \\
\hline & Neutral & 0.73 & 0.33 & 1.00 & 0.51 & 0.54 \\
\hline & Radial Deviation & 0.95 & 0.70 & 0.51 & 1.00 & 0.97 \\
\hline & Ulnar Deviation & 0.92 & 0.81 & 0.54 & 0.97 & 1.00 \\
\hline \multirow{7}{*}{ 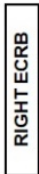 } & \multicolumn{6}{|c|}{ RIGHTECRB } \\
\hline & Position & Extension & Flexion & Neutral & Radial Deviation & Ulnar Deviation \\
\hline & Extension & 1.00 & 0.73 & 0.81 & 0.89 & 0.90 \\
\hline & \begin{tabular}{|l|} 
Flexion \\
\end{tabular} & 0.73 & 1.00 & 0.70 & 0.87 & 1.00 \\
\hline & Neutral & 0.81 & 0.70 & 1.00 & 0.64 & 0.81 \\
\hline & Radial Deviation & 0.89 & 0.87 & 1.00 & 1.00 & 0.94 \\
\hline & Ulnar Deviation & 0.90 & 0.94 & 0.81 & 0.94 & 1.00 \\
\hline
\end{tabular}

Figure 2. Left hand and right hand within flexor and extensor muscle activity wrist position Pearson correlation outputs. 
The correlations between muscles and between hands are shown in Figure 3. Correlations between the left ECRB and left FDS muscles were variable i.e. the correlation outputs consisted of both positive and negative associations that ranged between $r=-0.01$ (Poor) and $r=0.92$ (Very Strong). Correlations between the right ECRB and right FDS muscles were all positive and ranged between $r=0.01$ (Poor) and $r=0.85$ (Very Strong). Between hands the FDS (range: $r=-0.10$ to 0.84 ) and ECRB (range: $r=-0.02$ to 0.78 ) muscles respectively produced variable correlations with respect to wrist positions. There were significant differences between the left ECRB and left FDS $(P<0.005)$ for all wrist position associations. Similarly, the right ECRB and right FDS also showed significant differences $(P<0.029)$ for all wrist position associations. Conversely, no significant differences were observed for both the right FDS and left FDS wrist position associations together with the right ECRB and left ECRB wrist position associations respectively.

\begin{tabular}{|c|c|c|c|c|c|c|}
\hline \multirow{7}{*}{$\begin{array}{l}\text { 䍃 } \\
\text { w } \\
\text { w } \\
\text { w }\end{array}$} & \multicolumn{6}{|c|}{ LEFT FDS } \\
\hline & Position & Extension & Flexion & Neutral & Radial Deviation & Ulnar Deviation \\
\hline & Extension & 0.37 & 0.62 & 0.43 & 0.20 & -0.58 \\
\hline & \begin{tabular}{|l|} 
Flexion \\
\end{tabular} & -0.15 & 0.74 & 0.55 & -0.11 & -0.07 \\
\hline & Neutral & 0.01 & 0.77 & 0.92 & -0.12 & -0.38 \\
\hline & Radial Deviation & -0.01 & 0.62 & 0.35 & 0.70 & -0.21 \\
\hline & UInar Deviation & -0.62 & 0.20 & 0.36 & -0.23 & 0.36 \\
\hline \multirow{7}{*}{ 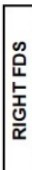 } & \multicolumn{6}{|c|}{ LEFT FDS } \\
\hline & Position & Extension & Flexion & Neutral & Radial Deviation & Ulnar Deviation \\
\hline & Extension & 0.15 & 0.63 & 0.76 & -0.46 & -0.53 \\
\hline & \begin{tabular}{|l|} 
Flexion \\
\end{tabular} & 0.33 & 0.80 & 0.72 & 0.25 & -0.10 \\
\hline & Neutral & 0.41 & 0.26 & 0.46 & -0.76 & -0.59 \\
\hline & Radial Deviation & 0.07 & 0.72 & 0.83 & -0.20 & -0.43 \\
\hline & Ulnar Deviation & 0.17 & 0.79 & 0.84 & -0.11 & -0.47 \\
\hline
\end{tabular}

\begin{tabular}{|c|c|c|c|c|c|c|}
\hline \multirow{7}{*}{ 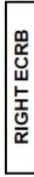 } & \multicolumn{6}{|c|}{ RIGHT FDS } \\
\hline & Position & Extension & Flexion & Neutral & Radial Deviation & Ulnar Deviation \\
\hline & Extension & 0.66 & 0.60 & 0.84 & 0.56 & 0.64 \\
\hline & Flexion & 0.32 & 0.85 & 0.34 & 0.38 & 0.53 \\
\hline & Neutral & 0.15 & 0.33 & 0.63 & 0.01 & 0.12 \\
\hline & Radial Deviation & 0.61 & 0.83 & 0.62 & 0.63 & 0.76 \\
\hline & Ulnar Deviation & 0.46 & 0.77 & 0.60 & 0.44 & 0.59 \\
\hline \multirow{7}{*}{ 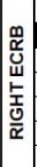 } & \multicolumn{6}{|c|}{ LEFT ECRB } \\
\hline & Position & Extension & Flexion & Neutral & Radial Deviation & Ulnar Deviation \\
\hline & Extension & 0.56 & 0.54 & 0.74 & 0.09 & -0.02 \\
\hline & Flexion & 0.55 & 0.49 & 0.56 & 0.47 & -0.10 \\
\hline & Neutral & 0.23 & 0.42 & 0.25 & -0.15 & -0.06 \\
\hline & Radial Deviation & 0.78 & 0.45 & 0.78 & 0.47 & -0.25 \\
\hline & Ulnar Deviation & 0.66 & 0.57 & 0.64 & 0.38 & -0.16 \\
\hline
\end{tabular}

Figure 3. Flexor and extensor muscle activity wrist position correlations between muscles and between hands.

\section{Discussion}

The scientific rationale of this study is a continuation of research conducted by Duque et al (1995) who carried out a similar study on the right hand only [30]. In addition, Sorbie et al (2018) highlighted the need to further investigate the reliability of the Myon 320 sEMG system (Myon AG, Switzerland) during dynamic contractions of the forearm muscles. To date there have been no major studies that have 
used the Myon 320 system (Myon AG, Switzerland) apparatus to validate the electrical activity of the forearm muscles. The aim of this study was to examine the reliability of the surface EMG signals from both the FDS and ECRB muscles of both left and right arms during individual, multi-planar MVC handgrip tasks.

Previous studies have reported using the right/dominant hand with the participant's upper arm being parallel with the trunk and the elbow being held at 90 degrees of flexion $[21,30,31]$. Furthermore, the study by Ngo and Wells (2016) recorded data from a natural power grip posture and then from induced wrist extension, flexion, pronator, supinator radial and ulnar deviation positions. In addition, Forman and colleagues measured forearm EMG with the wrist in extension, flexion and deviation in different humeral elevation angles [32]. This study has similarly been designed to analyse EMG output from the FDS and ECRB of both left and right arms with the elbow held at 90 degrees in neutral, flexion, extension, ulnar deviation and radial deviation. A study by Khalil et al (2016) showed that a significant increase in the Root Mean Square (RMS) of the Flexor Carpi Radialis and Flexor Carpi Ulnaris was observed when the elbow was positioned at 90 degrees as opposed to 0 degrees and 180 degrees [33].

A study by Agarwal et al (2018) includes both dominant and non-dominant hands with the wrist in flexion, extension, adduction and abduction [34]. However, their study produced sEMG data from the Flexor Carpi Radialis, Extensor Carpi Ulnaris, Flexor Digitorum Superficialis and the Extensor Digitorum Communis [34]. Our study performed different calculations in comparison to Agarwal et al's study, which was based on wrist angle, mean amplitude, EMG RMS as well as mean and median frequency [34]. This highlights a limitation in the recent experimental research that no studies have yet collected and reported data from both the FDS and ECRB muscles for both forearms over different wrist positions (Neutral, Flexion, Extension, Ulnar and Radial Deviation).

Throughout this study, repetitions of the test have been kept to a minimum and ambient room temperature standardized throughout the experimental protocol. To negate the effect of muscle 
fatigue or discomfort, each participant received a timed two-minute rest period per maximal effort. Petrofsky and Lind (1980) demonstrated that the testing environment should be kept at the same temperature and that the muscles of the participant should be free from fatigue [35]. Each effort was timed using a stopwatch for a five second period. This was then repeated with the opposing hand. This timing was based on the previous work of Duque et al (1995). Chaffin et al (2006) proved that maximum grip strength decreases dramatically for grip width lower than $60 \mathrm{~mm}$ and higher than $100 \mathrm{~mm}$ [36]. To accommodate this an adjustable pinch grip digital analyser (Medical Research Ltd (Leeds, UK)) was used to ensure an objective measurement of the participants grip strength.

All MVC trial data has been normalised by calculating as a percentage of the neutral mean. Duque et al (1995) pointed out that calibration of the force and EMG should be carried out in one neutral position for each participant and that other attempts may not be satisfactory in either flexion or extension positions as the EMG signal in flexion can show increases of up to $30 \%$ of the MVC in the same position [30]. This study has shown that $70 \%$ of the intra-session ICC results were in the good to excellent range. However, this has caused $15 \%$ of the results to be in the moderate $(0.5-0.75)$ range and $15 \%$ in the poor $(<0.5)$ range. These figures may be accredited to the variability in the extreme positioning of the hand and wrist during the MVC generation, the participants intrinsic competition across trials or indeed that the variability becomes relative with EMG and force values within stronger wrist positions. Results from the paired t-test highlight the use of the flexor/extensor muscle usage during the required positioning. This concurs with the study by Snijder et al (1987) who suggest that power grip is associated with the high loading of extensor muscles. Their study used both intramuscular and surface EMG outputs from tests which monitor the pushing effect of a finger on a platform as well as performing both gripping and pinching actions [1].

Pearson correlations were applied to the flexor and extensor muscles to better understand how strong the muscle activity firing pattern relationship is influenced by wrist position. This interest was further explored in this pilot study to ascertain firstly, whether associations existed within the FDS or ECRB 
when analysed independently with respect to wrist position. Secondly, it was important to learn whether differences were evident between the flexor and extensor muscle interactions at the different wrist positions. Our results produced both positive and negative correlations. Generally, the same hand and same muscle wrist position interactions presented with a trend. Positive correlations were found for the right hand FDS muscles, right ECRB muscles and right ECRB versus right FDS muscle interactions. Negative correlations which infer an inverse relationship showed the left FDS muscles, left ECRB muscles, left ECRB versus left FDS muscle interaction, right FDS versus left FDS muscle interaction, and right ECRB versus left ECRB interaction appeared to include at least two negative correlations with respect to wrist position. This information may be useful in terms of providing an improvement to current biomechanical hand modelling. The findings from our pilot study has scope for providing a clearer understanding of the synergistic muscle firing patterns and muscle recruitment strategies associated with the flexor and extensor muscles during fixed and dynamic wrist joint range of movement. Surgical procedures involving muscle transfers and forearm muscle reconstruction surgery may also find some merit in the interpretation of this finding. This reliability study has narrowly focused on a specific commercial device / EMG measuring system. Due to financial constraints and the inaccessibility to other EMG commercial devices our study was limited, and therefore our study design could not include comparisons against other EMG devices.

This study aimed to examine the reliability of surface EMG signals from both the Flexor Digitorum Superficialis and Extensor Carpi Radialis Brevis muscles of both left and right arms during an individual, static multi-planar maximum voluntary contraction handgrip task when using the Myon 320 system (Myon AG, Switzerland). Whilst the study produced good to fair reliability across the descriptive statistical outputs, further analysis and justification could have been included with the implementation of the collected MVC force data. Future directions from our laboratory include investigations on understanding the coupling of wrist movement and how these movements impact on the synergistic co-contractions of the flexor and extensor muscles during both static and dynamic related activities involving the wrist and fingers. Future trials may benefit from the inclusion of a 
custom splint or goniometer to ensure the exact wrist movement as well as facilitating the wrist positioning of participants who find difficulty in maintaining required positions. This may have had an impact on the ICC results. Previous studies have utilized many of the protocols included within this study, however, there are no precise testing guidelines available, thus making comparisons difficult.

\section{Conclusions}

This pilot study was undertaken to reliably test EMG signals using the Myon 320 sEMG System (Myon AG, Switzerland) taken from both the FDS and ECRB muscles of both left and right arms during an individual, static multi-planar MVC handgrip task. The study has highlighted that achieving reliability for normalized RMS SEMG during the tests is entirely possible. The study produced a fair reproducibility from the flexor and extensor muscles, which agrees with previous studies. The intrasession ICC analysis gave good to excellent results with the remaining results possibly benefitting by ensuring that future participants are prevented from knowing their force data during the trials and therefore preventing intrinsic competition. Wrist position correlations within and between the FDS and ECRB muscles may be influenced by hand dominance. Results from the study indicate that the data sets retrieved from all participants were reliably evaluated. With minor adjustments to the protocols used (removal of intrinsic competition), the findings from the study demonstrate that the methods and systems outlined here can be used reliably in future experimental and clinical trials.

Acknowledgements: The authors will like to thank Myon AG for their technical assistance during the early stages of the project. 


\section{References}

1. Snijders CJ, Volkers AC, Mechelse K, Vleeming A. Provocation of epicondylalgia lateralis (tennis elbow) by power grip or pinching. Medicine and science in sports and exercise. 1987;19(5):518-23.

2. Fess EE. The need for reliability and validity in hand assessment instruments. The Journal of Hand Surgery. 1986;11(5):621-3.

3. Nissenkorn A, Ben-Zeev B. Unilateral Rhythmic Hand Tapping in Rett Syndrome. Journal of Child Neurology. 2013;28(10):1210-4.

4. Innes E. Handgrip strength testing: A review of the literature. Australian Occupational Therapy Journal. 1999;46(3):120-40.

5. Newman DG, Pearn J, Barnes A, Young CM, Kehoe M, Newman J. Norms for hand grip strength. Archives of disease in childhood. 1984;59(5):453-9.

6. Tietjen-Smith T, Smith SW, Martin M, Henry R, Weeks S, Bryant A. Grip Strength in Relation to Overall Strength and Functional Capacity in Very Old and Oldest Old Females. Physical \& Occupational Therapy In Geriatrics. 2006;24(4):63-78.

7. Fry AC, Ciroslan D, Fry MD, LeRoux CD, Schilling BK, Chiu LZ. Anthropometric and performance variables discriminating elite American junior men weightlifters. Journal of Strength and Conditioning Research. 2006;20(4):861.

8. Budoff JE. The prevalence of rotator cuff weakness in patients with injured hands. The Journal of Hand Surgery. 2004;29(6):1154-9.

9. Shea J. The importance of grip strength. Journal of Strength and Conditioning Research. 2007;21(3):923-9.

10. Andrews JG, Youm Y. A biomechanical investigation of wrist kinematics. Journal of Biomechanics. 1979;12(1):83-93.

11. Hoffman DS, Strick PL. Step-Tracking Movements of the Wrist. IV. Muscle Activity Associated With Movements in Different Directions. Journal of Neurophysiology. 1999;81(1):319-33.

12. Hägg GM, Milerad E. Forearm extensor and flexor muscle exertion during simulated gripping work - An electromyographic study. Clinical Biomechanics. 1997;12(1):39-43.

13. Rashid H, Omar AR, Jaafar R, Abdullah SC, Ma'arof MIN, Fauzi WMSW, et al. Usage of Wireless Myon 320 Surface Electromyography (sEMG) System in Recording Motorcyclist Muscle Activities on Real Roads: A Case Study. Procedia Manufacturing. 2015;3(Ahfe):2566-73.

14. Bano F. EMG Investigations Regarding Handle Size , Grip Force and Stroke Rotation In Screw Driving. 2013(4):1-14.

15. Dickie JA, Faulkner JA, Barnes MJ, Lark SD. Electromyographic analysis of muscle activation during pull-up variations. Journal of Electromyography and Kinesiology. 2017;32:30-6.

16. Litzenberger S, Mally F, Sabo A. Biomechanics of elite recumbent handcycling: a case study. Sports Engineering. 2016;19(3):201-11.

17. Goh VH-H, Tong TY-Y, Lim C-L, Low EC-T, Lee LK-H. Effects of One Night of Sleep Deprivation on Hormone Profiles and Performance Efficiency. Military Medicine. 2001;166(5):427-31.

18. Cappaert TA. Review: Time of Day Effect on Athletic Performance: An Update. 1999.

19. Hoozemans MJM, Van Dieën JH. Prediction of handgrip forces using surface EMG of forearm muscles. Journal of Electromyography and Kinesiology. 2005;15(4):358-66.

20. Chen HM, Chen C, Hsueh IP, Huang SI, Hsieh CL. Test-Retest Reproducibility and Smallest Real With Stroke. Neurorehabilitation and Neural Repair. 2009;23(17):435-40.

21. Sorbie GG, Williams MJ, Boyle DW, Gray A, Brouner J, Gibson N, et al. Intra-session and interday reliability of the Myon 320 electromyography system during sub-maximal contractions. Frontiers in Physiology. 2018;9(MAR):1-7.

22. Incel NA, Ceceli E, Durukan PB, Rana Erdem H, Rezan Yorgancioglu Z. Grip Strength: Effect of Hand Dominance. Singapore Medical Journal. 2002;43(5):234-7. 
23. Sorbie GG, Hunter HH, Grace FM, Gu Y, Baker JS, Ugbolue UC. An electromyographic study of the effect of hand grip sizes on forearm muscle activity and golf performance. Research in Sports Medicine. 2016;24(3):222-33.

24. Basmajian JV. Biofeedback: Principles and practice for clinicians. 1989.

25. Akoglu H. User's guide to correlation coefficients. Turk J Emerg Med. 2018;18(3):91-3.

26. Chan YH. Biostatistics 104: correlational analysis. Singapore Med J. 2003;44(12):614-9.

27. Atkinson G, Nevill AM, Edwards B. Communications to the Annual Conference of the British Association of Sport and Exercise Sciences ( BASES ). Journal of Sport Sciences. 1999;17:3-70.

28. Tavakol M, Dennick R. Making sense of Cronbach's alpha. International journal of medical education. 2011;2:53.

29. Richardson JT. Eta squared and partial eta squared as measures of effect size in educational research. Educational Research Review. 2011;6(2):135-47.

30. Duque J, Masset D, Malchaire J. Evaluation of handgrip force from EMG measurements. Applied Ergonomics. 1995;26(1):61-6.

31. Ngo BPT, Wells RP. Evaluating protocols for normalizing forearm electromyograms during power grip. Journal of Electromyography and Kinesiology. 2016;26:66-72.

32. Forman DA, Baarbé J, Daligadu J, Murphy B, Holmes MWR. The effects of upper limb posture and a sub-maximal gripping task on corticospinal excitability to muscles of the forearm. Journal of Electromyography and Kinesiology. 2016;27:95-101.

33. Khalil A, Raoof N, Battecha K. Effect of Different Elbow Angles Positions on Hand Grip Strength and Myoelectrical Activity of Wrist Flexors in Healthy Subjects. International Journal of Therapies and Rehabilitation Research. 2016;5(5):198-.

34. Agarwal R, Kumar H, Singh S. EMG and Grip Force correlation at varying wrist angles for Dominant vs. non-dominant hand. 2018 IEEE International Students' Conference on Electrical, Electronics and Computer Science, SCEECS 2018. 2018:1-4.

35. Petrofsky JS, Lind AR. The influence of temperature on the amplitude and frequency components of the EMG during brief and sustained isometric contractions. European Journal of Applied Physiology and Occupational Physiology. 1980;44(2):189-200.

36. Chaffin DB, Andersson GB, Martin BJ. Occupational biomechanics: John wiley \& sons; 2006. 
$\underline{\text { Tables }}$

Table 1. Comparative percentage against Neutral.

\begin{tabular}{lllll}
\hline Wrist Position & Left FDS & Right FDS & Left ECRB & Right ECRB \\
\hline Extension & $79.86 \pm 29.32$ & $101.36 \pm 46.24$ & $87.64 \pm 25.23$ & $97.89 \pm 46.03$ \\
Flexion & $95.54 \pm 37.50$ & $115.48 \pm 57.38$ & $117.40 \pm 34.22$ & $128.74 \pm 73.88$ \\
Radial Deviation & $72.78 \pm 38.87$ & $99.57 \pm 57.53$ & $98.66 \pm 44.45$ & $111.97 \pm 60.99$ \\
Ulnar Deviation & $100.96 \pm 44.59$ & $122.11 \pm 57.24$ & $120.65 \pm 52.42$ & $134.92 \pm 69.00$ \\
Neutral & $100.00 \pm 0.00$ & $100.00 \pm 0.00$ & $100.00 \pm 0.00$ & $100.00 \pm 0.00$ \\
\hline
\end{tabular}

Table 2. Muscle activation outputs and reliability measurements of the ECRB and FDS muscles in five wrist positions.

\begin{tabular}{lllllll}
\hline $\begin{array}{l}\text { Muscle } \\
\text { Activation }\end{array}$ & $\begin{array}{l}\text { Reliability } \\
\text { Measurement }\end{array}$ & Extension & Flexion & Neutral & $\begin{array}{l}\text { Radial } \\
\text { Deviation }\end{array}$ & $\begin{array}{l}\text { Ulnar } \\
\text { Deviation }\end{array}$ \\
\hline Left FDS & SD & $29.32 \%$ & $37.50 \%$ & $100.00 \%$ & $38.87 \%$ & $44.59 \%$ \\
& SEM & $3.31 \%$ & $4.24 \%$ & $11.31 \%$ & $4.39 \%$ & $5.04 \%$ \\
& CV & 0.17 & 0.48 & 0.38 & 0.36 & 0.25 \\
Right FDS & SD & $49.43 \%$ & $61.34 \%$ & $100.00 \%$ & $61.51 \%$ & $61.20 \%$ \\
& SEM & $5.59 \%$ & $6.94 \%$ & $11.31 \%$ & $6.95 \%$ & $6.92 \%$ \\
& CV & 0.85 & 0.79 & 0.59 & 1.00 & 0.86 \\
Left ECRB & SD & $26.97 \%$ & $36.58 \%$ & $100.00 \%$ & $47.52 \%$ & $56.04 \%$ \\
& SEM & $3.05 \%$ & $4.14 \%$ & $11.31 \%$ & $5.37 \%$ & $6.34 \%$ \\
& CV & 0.39 & 0.26 & 0.34 & 0.43 & 0.27 \\
Right ECRB & SD & $52.11 \%$ & $70.09 \%$ & $100.00 \%$ & $56.48 \%$ & $61.72 \%$ \\
& SEM & $5.89 \%$ & $7.93 \%$ & $11.31 \%$ & $6.39 \%$ & $6.98 \%$ \\
& CV & 0.51 & 0.72 & 0.64 & 0.52 & 0.59 \\
\hline
\end{tabular}

Standard Deviation (SD), Standard Error of Measurement (SEM) and Coefficient of Variation (CV). 


\section{Figure Captions}

Figure 1. (a) Pinch / Grip Digital Analyzer Device (b) Pictorial illustration of a subject using Pinch / Grip Digital Analyzer Device with forearm secured in a customized arm rig.

Figure 2. Left hand and right hand within flexor and extensor muscle activity wrist position Pearson correlation outputs.

Figure 3. Flexor and extensor muscle activity wrist position correlations between muscles and between hands. 


\section{Figures}

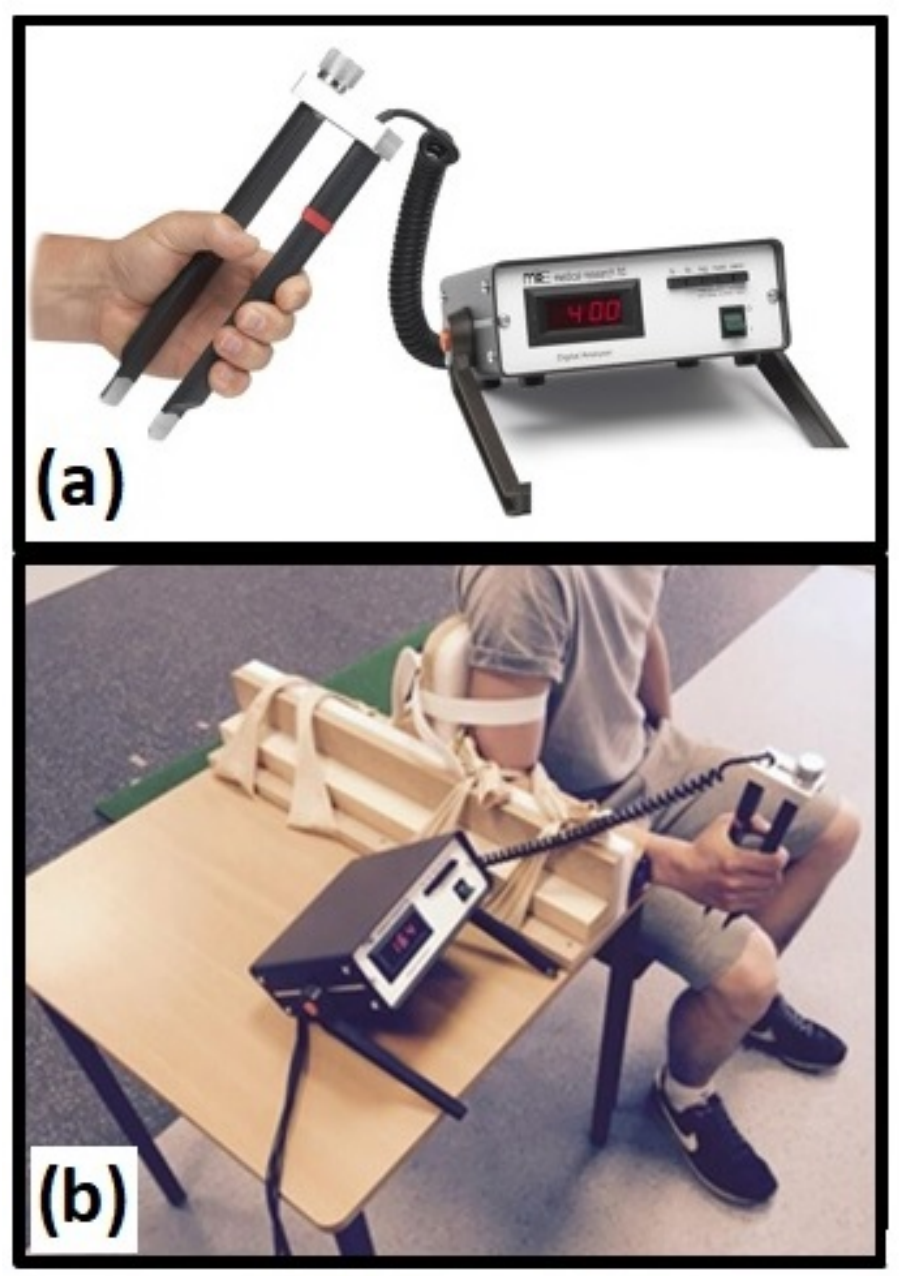

Figure 1.

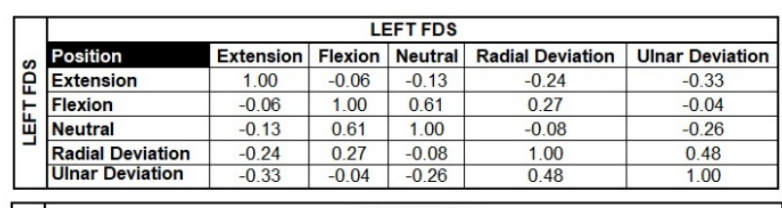

\begin{tabular}{|c|c|c|c|c|c|c|}
\hline \multirow{7}{*}{$\begin{array}{l}\text { on } \\
\underline{4} \\
\frac{\underline{0}}{\alpha} \\
\frac{0}{\alpha}\end{array}$} & \multicolumn{6}{|c|}{ RIGHT FDS } \\
\hline & Position & Extension & Flexion & Neutral & Radial Deviation & Ulnar Deviation \\
\hline & Extension & 1.00 & 0.60 & 0.73 & 0.95 & 0.92 \\
\hline & Flexion & 0.60 & 1.00 & 0.33 & 0.70 & 0.81 \\
\hline & Neutral & 0.73 & 0.33 & 1.00 & 0.51 & 0.54 \\
\hline & Radial Deviation & 0.95 & 0.70 & 0.51 & 1.00 & 0.97 \\
\hline & UInar Deviation & 0.92 & 0.81 & 0.54 & 0.97 & 1.00 \\
\hline \multirow{7}{*}{ 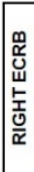 } & \multicolumn{6}{|c|}{ RIGHT ECRB } \\
\hline & Position & Extension & Flexion & Neutral & Radial Deviation & Ulnar Deviation \\
\hline & Extension & 1.00 & 0.73 & 0.81 & 0.89 & 0.90 \\
\hline & Flexion & 0.73 & 1.00 & 0.70 & 0.87 & 1.00 \\
\hline & Neutral & 0.81 & 0.70 & 1.00 & 0.64 & 0.81 \\
\hline & Radial Deviation & 0.89 & 0.87 & 1.00 & 1.00 & 0.94 \\
\hline & Ulnar Deviation & 0.90 & 0.94 & 0.81 & 0.94 & 1.00 \\
\hline
\end{tabular}

Figure 2. 


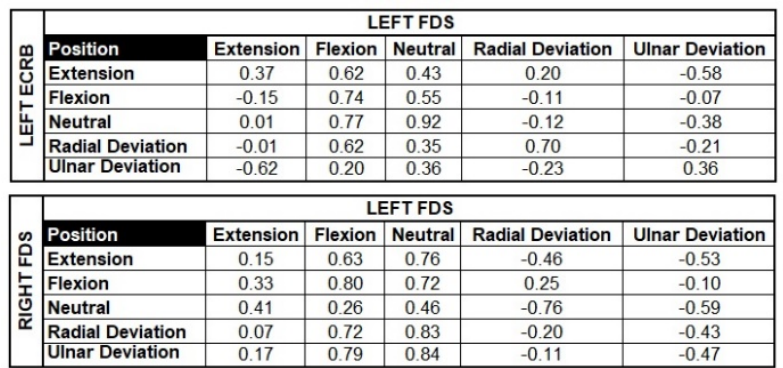

\begin{tabular}{l} 
Radial Deviation \\
\hline Ulnar Deviation
\end{tabular}

\begin{tabular}{|c|c|c|c|c|c|}
\hline \multicolumn{6}{|c|}{ RIGHT FDS } \\
\hline Position & Extension & Flexion & Neutral & \begin{tabular}{|l} 
Radial Deviation \\
\end{tabular} & Ulnar Deviation \\
\hline Extension & 0.66 & 0.60 & 0.84 & 0.56 & 0.64 \\
\hline Flexion & 0.32 & 0.85 & 0.34 & 0.38 & 0.53 \\
\hline Neutral & 0.15 & 0.33 & 0.63 & 0.01 & 0.12 \\
\hline Radial Deviation & 0.61 & 0.83 & 0.62 & 0.63 & 0.76 \\
\hline Ulnar Deviation & 0.46 & 0.77 & 0.60 & 0.44 & 0.59 \\
\hline \multicolumn{6}{|c|}{ LEFT ECRB } \\
\hline Position & Extension & Flexion & Neutral & Radial Deviation & Ulnar Deviation \\
\hline Extension & \begin{tabular}{|l|l|}
0.56 \\
\end{tabular} & 0.54 & 0.74 & 0.09 & \begin{tabular}{|l|}
-0.02 \\
\end{tabular} \\
\hline Flexion & 0.55 & 0.49 & 0.56 & 0.47 & -0.10 \\
\hline Neutral & 0.23 & 0.42 & 0.25 & -0.15 & -0.06 \\
\hline Radial Devi & 0.78 & 0.45 & 0.78 & 0.47 & -0.25 \\
\hline Ulnar Deviation & 0.66 & 0.57 & 0.64 & 0.38 & -0.16 \\
\hline
\end{tabular}

Figure 3. 